\title{
Conductivity and Length Relationships in Heat-Flow Transducer Performance
}

\author{
Donald J. Portman
}

\begin{abstract}
In recent years many investigators have used thermoelectric heat-flow transducers to measure heat conduction in natural soil. If the transducer has thermal properties different from those of the soil it will interfere with the natural heat transfer and an error in measurement will occur. An analysis of the error for a transducer placed in an infinite medium in steady-state conditions is made in terms of two non-dimensional parameters: (1) the ratio of transducer conductivity to soil conductivity, and (2) the ratio of transducer thickness to transducer width. An exponential relationship is postulated on the basis of physical considerations and is verified by the results of an electrical analogy experiment for a square transducer having a thickness-width ratio of 0.125 for conductivity ratios from about 0.1 to 2.2. An error of -70 pct was found for a conductivity ratio of 0.1 and an error of +5 pct for a conductivity ratio of 2.2. The results are used to estimate error in measurements made during the Great Plains Turbulence Field Program.
\end{abstract}

Introduction - A heat-flow transducer, or heatHow meter, is a device for the direct measurement of heat transfer in one dimension. It usually consists of a thin sheet or plate of insulating material fitted with thermocouple junctions. The latter are arranged so that they form a thermopile having an output proportional to the temperature gradient across the plate. In steady conditions, thereiore, the thermopile output is a direct measure of heat conduction through the plate.

In studying evaporation from natural soil, snow, or vegetation-covered surfaces by means of the energy balance a heat-flow transducer may be used to determine heat conduction in the soil. lise of such a device in soil was first reported by Fulkenberg [1930] who attempted to use a circular Woods metal plate by placing it directly on the soil surface. Dunkle [1940] described an improved 'heat meter' made of Bakelite and having about 480 thermocouple junctions placed in a plate oneeighth inch thick and four and one-half inches siquare. He suggested that it be used in soil as well as in other media to measure heat transfer. Dacon [1950] described two transducers for soil heat-transfer measurements. He showed how they could be used by placing them one centimeter or less beneath the soil surface. Since that time tansducers of the type described by Dunkle have been used by many investigators. Reference may be made, for example, to Brooks and Kelly [1951], Gerhardt [1957], Portman [1957], and Rhoades [1057].

Heat-flow transducers have been used for many ither purposes, particularly for measuring heat transfer in building walls. They might be used, as well, in dams and other hydrological structures wherever it is desired to measure heat transfer.

Although the use of a transducer is perhaps the simplest and most convenient method of measuring soil heat transfer it is not always without difficulty. An important problem arises from the fact that the transducer acts as a barrier to the movement of soil moisture. Transducers made by Gier and Dunkle for use in soil had a rectangular grid of 25 holes, $\frac{3}{16}$ inches in diameter, equally spaced over the $4 \frac{1}{2} \times 4 \frac{1}{2}$ inch plate. The holes permitted vertical movement of water and water vapor. It is probable that, for a solid transducer, the barrier effect would decrease as the horizontal dimension of the plate is decreased. Nevertheless, transducers have been found to give relatively accurate information for energy-balance studies in moderately dry soil and for heat transfer at depths where vertical movement of water is negligible.

Another difficuity arises if the thermal properties of the transducer are significantly different from those of the soil. For steacly-state conditions, only thermal conductivity and length relationships enter the problem. If the conductivity of the transducer is not equal to that of the soil, the soil heat-flow pattern will be distorted. For a given conductivity inequality the amount of distortion will depend on the width and the thickness of the transducer and the depth of placement in the soil. If there is distortion, the heat flow through the transducer will not equal that in the undisturbed soil for an equal area. It is the purpose of this 
analysis to determine the effect of conductivity and length relationships in the performance of heat-flow transducers.

Theoretical considerations-The following discussion and conclusions apply to a square transducer in steady-state conditions. It is assumed, moreover, that if the two conductivities are equal, no discontinuity of conductivity exists at the soiltransducer interfaces, and if they are not equal an abrupt discontinuity exists, that is, there is no 'contact resistance.'

With these assumptions it can be physically reasoned that the ratio $X$ of the transducer heat flow $q_{t}$ to the undisturbed soil heat flow $q_{s}$ will be determined by the soil conductivity $k_{s}$, the transducer conductivity $k_{t}$, the transducer width (= length) $W$, the transducer thickness $L$, and the depth $D$ of transducer placement. Thus

$$
X=q_{t} / q_{s}=\phi_{1}\left(k_{t}, k_{s}, W, L, D\right)
$$

in which $\phi_{1}$ is an unknown function. By inspection it is seen that the five variables can be combined in three non-dimensional ratios for convenience of analysis, and (1) can be written

$$
X=\phi_{2}\left(k_{t} / k_{s}, L / W, D / W\right)
$$

It is apparent that if $k_{t}<k_{s}$ then $q_{t}<q_{s}$ and if $k_{t}>k_{s}$ then $q_{t}>q_{s}$. In the former case the transducer offers more resistance to heat flow than does an equal thickness of soil. There will be a tendency, consequently, for heat to flow around the transducer. In the latter case the opposite condition exists so that more heat tends to flow through the transducer than through an equal thickness of soil. Furthermore, an increase in $L / W$ can be thought of as an increase in $L$ holding $W$ constant. Hence, it is obvious that the distortion, as indicated by the deviation of $X$ from unity, will increase with an increase in thickness $L$. On the other hand, a decrease in $L / W$ can be thought of as a decrease in $L$ holding $W$ constant, and it becomes clear that this would decrease the degree of distortion.

If it is assumed that at the soil surface there exists a uniform heat source or sink, uninfluenced by the nearness of the transducer, it can be reasoned that a decrease in depth will decrease any existing distortion, other factors being constant. A uniform source or sink at the surface implies that the flow lines there are parallel and vertical, that is, perpendicular to the surface. Distortion at the transducer implies that as the transducer is approached from the surface the flow lines tend either to concentrate toward the center of the transducer or to deflect away from it. It can be imagined that as the depth is decreased there can be less deflection, or less concentration, merely because more curvature in flow lines will be required to effect the same distortion. For $k_{t}<k_{s}$, as with the case for a wide transducer, resistance for heat to flow through the transducer would be less effective since an increase in curvature means an increase of the path to flow around the transducer. Similar, but converse, reasoning would explain the opposite situation.

The model of a uniform source or sink at the surface seems somewhat unrealistic, however. If a transducer for which $k_{t} / k_{s} \neq 1$ were very near the surface, the flow pattern distortion would alter the surface temperature and a uniform source or sink could no longer be assumed to exist. Actually, the energy transfers at the surface could then be expected to be different and the situation would be dependent on external conditions. It is difficult to estimate these effects in rendering the above model invalid.

Because of the last-mentioned difficulty and because a solution of the problem would have application without consideration of the dependency on depth it seems reasonable to consider the case for a transducer placed in an infinite medium for a first analysis. The equation to be solved then is

$$
X=\phi_{3}\left(k_{t} / k_{a}, L / W\right)
$$

Considerable insight on the problem may be gained by establishing the limits of $X$ for various values of $k_{t} / k_{s}$ for any $L / W$. The following inequalities establish the envelope of a family of curves for all values of $L / W:$ For $k_{t}>k_{s}, q_{t}>$ $q_{s}$, and $\Delta T / L<G, X>1$ and $(\Delta T / L) / G<1$, in which $\Delta T$ is the average temperature difference across the transducer and $G$ the vertical temperature gradient in undisturbed soil. Since

$$
X=\frac{k_{t}}{k_{s}} \frac{\Delta T / L}{G}, \quad X<\frac{k_{t}}{k_{s}}
$$

Conversely, for

$$
k_{t}<k_{s}, \quad X>k_{t} / k_{s}
$$

The cross-hatched areas in Figure 1 show the regions in which the curves must lie for all values of $L / W$. Note that all curves must pass through the points $X=k_{t} / k_{s}=0$ and $X=k_{t} / k_{s}=1$.

The form of a curve within this envelope may be explored with the following reasoning.

From (3) and the defining relationships of $q t$ 
and $q_{s}$

$$
\frac{\Delta T / L}{G}=\phi_{4}\left(k_{t} / k_{x}, L / W\right)
$$

and as $k_{i} \rightarrow 0$

$$
\frac{\Delta T / L}{G}=\phi_{4}(0, L / W)
$$

The latter function, $\phi_{4}(0, L / W)$, must equal or be greater than unity and it must approach unity as $\mathrm{L} / \mathrm{W}$ approaches infinity. Since in reality $\mathrm{L} / \mathrm{W}$ ratios for transducers are always smaller than, at most, 0.1 , it may be established that $\phi_{4}(0,0.1) \equiv$ $d / G>1$. It follows, then, that

$$
X=\frac{k_{t} \Delta T / L}{k_{s} G}<\frac{k_{t} A}{k_{s} G}
$$

in which $A$ has an undetermined value.

Similarly, for $k_{t}$ approaching infinity

$$
X=\phi_{5}(\infty, L / W) \geqq 1
$$

and in this case as $L / W \rightarrow 0, X \rightarrow 1$, and as $L / W$ approaches its maximum value 0.1

$$
X=\phi_{5}(\infty, 0.1) \equiv \mathrm{C}
$$

in which $C$ has an undetermined value.

Hence the areas of Figure 1 are restricted by the above considerations to those indicated in Figure 2. The lines $X=\left(k_{t} / k_{s}\right)(A / G)$ and $X=C$ were arbitrarily placed.

The problem remains of finding the function $\phi_{3}$ in (3) with the conditions that as

$$
k_{t} / k_{s} \rightarrow 0, \quad X \rightarrow 0
$$

and as

$$
k_{t} / k_{s} \rightarrow 1, \quad X \rightarrow 1
$$

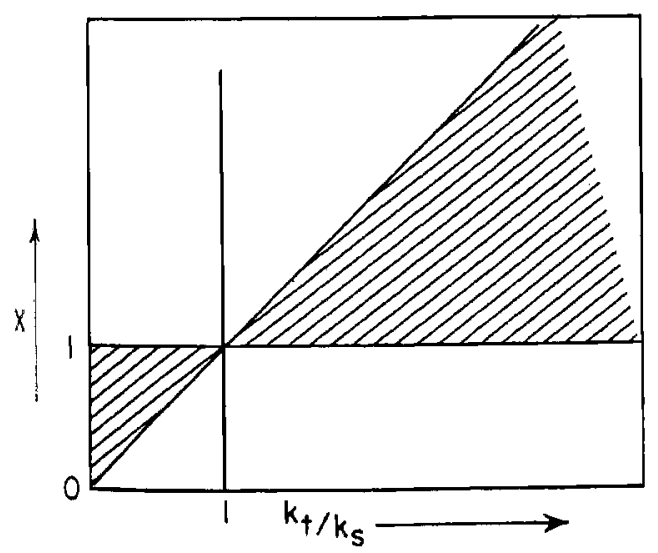

FIG. 1 - Flux ratio limits

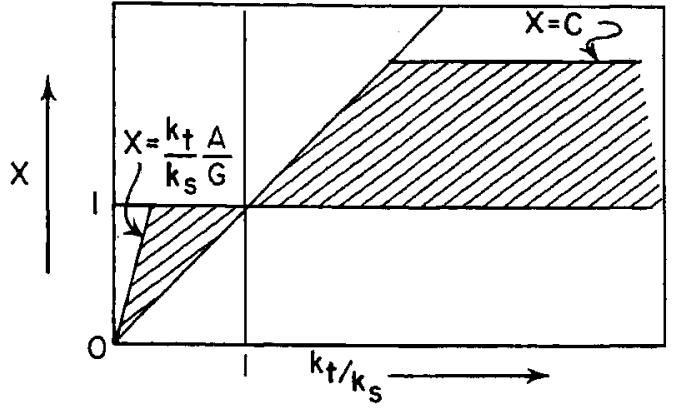

FIG. 2-Restricted flux ratio limits

The two conditions immediately suggest that $\phi_{3}$ is an exponential function. Hence, taking logarithms, one may write (3) as

$$
\ln X=\phi_{6}\left(k_{t} / k_{s}, L / W\right)
$$

or

$$
\ln X=\sum_{n=0}^{\infty}(L / W)^{n} \psi_{n}\left(k_{t} / k_{k}\right)
$$

with $\psi$ representing another function. For values of $L / W$ much less than one, powers of $L / W$ may be neglected for a first approximation. Thus

$$
\ln X \approx \psi_{0}\left(k_{t} / k_{s}\right)+L / W \psi_{1}\left(k_{t} / k_{s}\right)
$$

but as

$$
\begin{aligned}
L / W & \rightarrow 0 \\
X & \rightarrow 1 \\
\ln X & \rightarrow 0 \\
\text { and } \psi_{0}\left(k_{t} / k_{s}\right) & \rightarrow 0
\end{aligned}
$$

therefore

$$
\ln X \approx L / W \psi_{1}\left(k_{t} / k_{n}\right)
$$

or

$$
X \approx e^{L / W \psi_{1}\left(k_{i} t / k_{k}\right)}
$$

From (7) it was found that the function could be expressed as in the following equation

$$
X=e^{a_{L} / W\left(1-k_{s} / k_{l}\right)}
$$

in which $a$ is assumed to be a constant greater than zero.

Experimental verification-An electrical analogy experiment was used in an attempt to verify the form of (10) and to evaluate $a$. The experiment consisted of submerging a model of a heat-flow transducer in a tank of water in which a uniform and steady alternating current potential was maintained. The electrical resistance of the model 
was varied and, in effect, the current flow through the model was compared to the undisturbed flow in the tank.

This method of verifying (10) was undertaken mainly because the necessary electrical equipment could be readily obtained on loan from the Department of Civil Engineering of Johns Hopkins University. It seemed admirably suited to the problem because of the geometrical and conductivity relationships involved. The analytic approach for the three dimensional case appeared to be particularly cumbersome without an electronic computer since lengthy approximation methods would be required. Furthermore, an analogy method with resistance networks did not seem to offer a very direct approach.

The experimental technique and the electrical system employed have been described by $L i$ and others [1954] who used the same equipment to solve a water-flow problem. The physical system consisted of three components: (1) an alternating current source of about $800 \mathrm{c} / \mathrm{s},(2)$ a combined potentiometer and Wheatstone bridge arrangement with earphones and probe, and (3) the electrolytic tank, its electrodes and the model.

Alternating current is required for an experiment of this type to avoid deposition of the electrolyte at the electrodes. An audio frequency is employed so that the experimenter can use earphones instead of a galvanometer, enabling him to locate equipotential lines in the electrical field by merely listening for a minimum tone. The potentiometer circuit was used in this case to measure the average potential difference between the two sides of the transducer model and to determine the potential drop over an equal interval in an undisturbed portion of the tank. The Wheatstone bridge served to evaluate the total resistance of the model and the resistance of the water in the tank.

The potentiometer and bridge were so arranged that a single switch changed the whole system from one for measuring resistance to one for measuring potential, or vice versa. The audio frequency source and the earphones and probe were used for both systems. Since the circuit diagrams have been published [ $\mathrm{Li}$ and others, 1954, pp. $807,808]$ the electrical system will not be further discussed.

An ordinary rectangular glass aquarium was obtained for the electrolytic tank. It was approximately $25 \times 50 \mathrm{~cm}$, and $30 \mathrm{~cm}$ deep. The two ends were fitted with flat copper sheets to form electrodes covering each of the inner end surfaces. The tank was filled with tap water which had enough impurities to form an electrolyte of a convenient resistance for the ranges of the bridge and the potentiometer.

Because of symmetry of the flow pattern through and around an imbedded transducer, a model was constructed to represent a quarter section of the transducer. It was attached to the wall of the tank midway between the two electrodes, with its sides parallel to the tank electrodes and its upper edge flush with the water surface. The dimensions of the model were $10 \times 10 \times 2.5 \mathrm{~cm}$ representing, therefore, a transducer with an $\mathrm{L} / \mathrm{W}$ ratio of 0.125 . Essentially, the model was a rectangular containter to hold water of different resistivities. It was made of non-conducting materials; the edge material was $\frac{1}{8}$ inch thick and that of the two sides was $\frac{1}{16}$ inch thick. Each side was perforated with 225 holes in a rectangular grid. A brass machine screw was then turned part way into each hole and the protruding screw ends were sheared off. Consequently electricity could flow perpendicularly but not laterally through the model sides.

The model was filled first with a sample of the tank water and the area around the model was probed to evaluate distortion in the potential field caused by the walls of the model. It was found that the distortion could not be detected with the electrical system employed and it was assumed, consequently, that the effect of the model surfaces could be neglected.

Various concentrations of tap water, distilled water, and salt water were used in the model to obtain the desired conductivity ratios. In each case the potential drop across the sides of the model was determined by measuring the potential at 25 points on each side. Since the equipotential surfaces formed curves at their intersections with the model sides, the average potential of a side could not be determined directly. The potential values were plotted on graph paper, equipotential lines were drawn and the average for the area 1 was graphically determined from the pattern formed.

Resistivities of the tank water and of the variow: model solutions were determined by placing samples in a non-conducting cylindrical test cell $10 \mathrm{~cm}$ in length and $5 \mathrm{~cm}$ in diameter, the ends of which were closed by tightly fitting copper diks which served as electrodes. The resistance $R$ of thit cell was measured with the Wheatstone bridge and the resistivity $r$ of each sample determined bs

$$
r=R(S / l) \quad(\text { ohm } \mathrm{cm})
$$


in which $S$ is the cross sectional area of the cell and $l$ its length.

The ratio of the current flow through the model to the current flow for an equal area in the undisturbed tank water was calculated with the relationship

$$
I_{m} / I_{\iota}=\left(r_{t} / r_{m}\right)\left(E_{m} / E_{i}\right)
$$

in which

$I_{m}=$ current flow through model

$I_{i}=$ current flow through an equal area in the tank

$r_{m}=$ resistivity of model water

$n_{1}=$ resistivity of tank water

$E_{m}=$ potential drop across model

$E_{t}=$ potential drop across distance in tank

Eq. (12) follows from Ohm's law after substitution with the relationship of (11) above. $I_{m} / I_{t}$ is the analogue of $X$ in (10) and $r_{t} / \gamma_{m}$ the analogue of $k_{t} / k_{8}$.

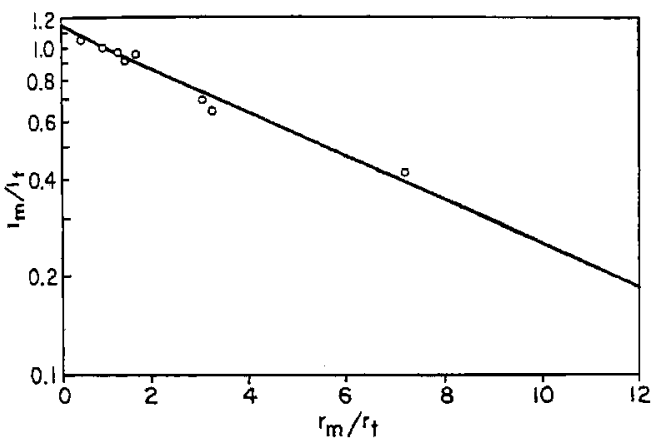

FIG, 3 - Relationship between current flow ratio and resistivity ratio (ordinate on logarithmic scale)
The following data were obtained:

\begin{tabular}{l|l}
\hline$r / r_{m}$ & $I_{m} / I_{t}$ \\
\hline 0.14 & 0.417 \\
0.30 & 0.640 \\
0.325 & 0.689 \\
0.60 & 0.954 \\
0.67 & 0.910 \\
0.73 & 0.970 \\
1.00 & 1.000 \\
2.23 & 1.060 \\
\hline
\end{tabular}

Because an exponential relationship was predicted between $X$ and $k_{s} / k_{t}$, their electrical analogues were plotted in semi-logarithmic co-ordinates as shown in Figure 3. It is seen that the experimental points approximate a straight line as expected. The line shown was placed by visual estimation with the requirement that it pass through the point $1.0,1.0$. Its equation is

$$
\text { In }\left(I_{m} / I_{t}\right)=b\left(1-\tau_{m} / r_{t}\right)
$$

in which $b$ is a constant found to be approximately 0.15. Eq. (13), rewritten as an exponential function, becomes

$$
I_{m} / I_{t}=e^{b\left(1-r_{m} / r t\right)}
$$

which is the analogy of (10). Consequently, $b=a(L / W)$ and substituting $L / W=0.125$ for the model used in the experiment, $b=0.15$, and $a=1.2$. Thus, (10) becomes

$$
X=e^{1.2(L / W)\left(1-k_{x} / k_{t}\right)}
$$

Figure 4 shows the curve of (15) for $L / W=$ 0.125 and the experimental points obtained. This

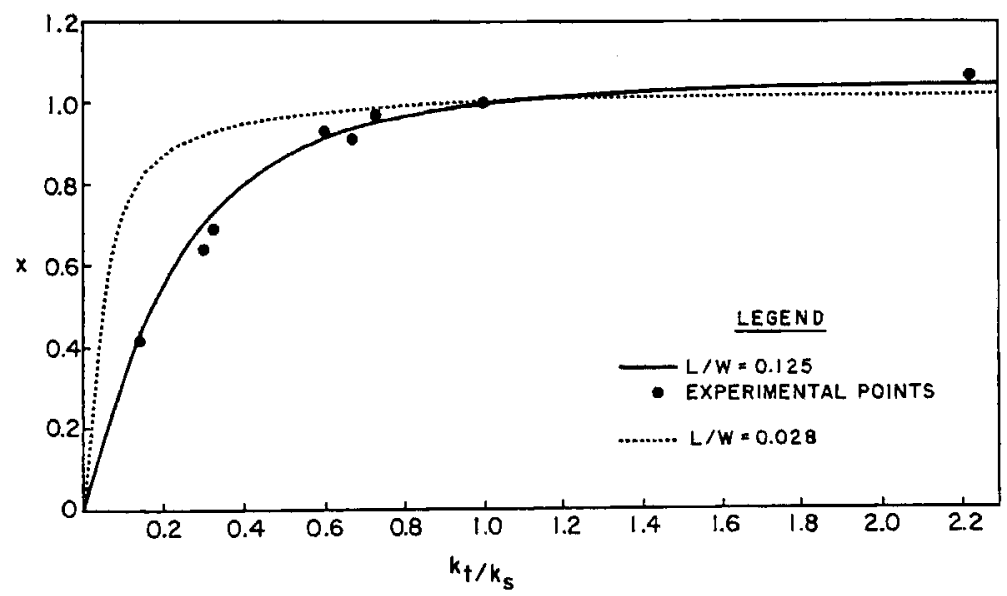

FIG. 4 - Relationship between heat flow ratio and conductivity ratio: $X=e^{a(L / W)\left[1-\left(k_{\mathrm{a}} / k_{t}\right)\right]}$ 
is, of course, merely another representation of the information shown in Figure 3, but it shows also the curve in relationship to the limiting areas discussed earlier.

Application of results-Shown also in Figure 4 is the curve for an $L / W$ ratio 0.028 , an appropriate value for a heat-flow transducer used during the Great Plain Turbulence Field Program at O'Neill, Nebraska [Portman, 1957]. Since the transducer had steel cover plates approximately $\frac{1}{32}$ inch thick over the $\frac{3}{64}$ inch Bakelite element, the computed curve might not be valid for this case. Steel has a conductivity about 100 times that of the Bakelite, consequently the flow-pattern distortion caused by this transducer would be somewhat different from that of the simple model.

If an overall effective conductivity is used for the transducer, the curve might have meaning. An effective conductivity $k_{e}$ was computed using the following equation

$$
k_{z}=\frac{L_{1}+L_{2}}{L_{1} / k_{1}+L_{2} / k_{2}} \approx 0.0016 \mathrm{cgs} \text { units }
$$

in which the subscripts 1 and 2 refer to Bakelite and steel respectively. Buettner and others [1957] measured the soil thermal conductivity at the O'Neill test site and have reported that the values at $2.5 \mathrm{~cm}$ depth ranged from $0.8 \times 10^{-8}$ to $1.3 \times$ $10^{-3}$ for the observation days for which transducer data are given by Portman [1957]. The corresponding $k_{t} / k_{s}$ ratios are approximately 2 and 1.2. If the curve is correct for this transducer and if the empirical constant $a=1.2$ is applicable for an $L / W$ ratio of 0.028 , Figure 4 shows that the ONeill data should have less than +2 per cent error due to flow pattern distortion.

Conclusions-Figure 4 reveals that for conductivity ratios between 0.8 and 2.0 an error of less than five per cent would be involved when a transducer having a thickness-width ratio of 0.125 is used. If the transducer thickness is $\frac{3}{64}$ inch, the standard thickness of a type of transducer commercially available, it could be as small as $\frac{1}{2}$ inch square and have distortion errors no larger than those indicated by the solid line in Figure 4. Such a transducer would be much more satisfactory for use in wet soil than would the larger de- vices currently being used, because of the moisturs barrier problem previously discussed. Severa? could be used at one depth to obtain more representative measurements and they could, presum. ably, be connected in series in order to increase the electrical output if necessary.

The analysis shows, further, that if a transiucer with an $L / W$ ratio on the order of 0.1 is to be used in soil whose thermal conductivity is likely change with time it is best to have the transduce: conductivity somewhat larger than that of the soil since the error is small and only slowly chang. ing between conductivity ratios of 1 and 2 .

Acknowledgments - This contribution is based on chapter of a dissertation submitted in partial fulfilmest of the requirements for the degree of Doctor of $P$ L: losophy at Johns Hopkins University. The author indebted to George S. Benton for many suggestions axd for helpful criticism in the analysis of the problem.

\section{REFERENCES}

BRoOKS, F. A., AND C. F. KEILY, Instrumentation for recording microclimatological factors, Trows. A war. Geophys. Union, 32, 833-848, 1951.

Buettrer, K., R. C. Staley, and B. E. Moser, Thermal conductivity, Exploring the Almospheres First Mile (H. Lettau and B. Davidson, ed.), Perga. mon Press, pp. 29-38, 1957.

DEACON, E. I., The measurement and recording of the heat flux into the soil, Q.J.R.Met. Soc., 76, 479-483, 1950.

DunkLe, R. V., Heat meters, Bul. Amer. Met. Sor, 21, $116-117,1940$.

FaIkenberg, G., (no title), Met. Zs. 47, 154-155, 1930.

GerhardT, J. R., Albrecht and Gier and Dunkle heat flow meters, Exploring the Atmosphere's First II he (H. Lettau and B. Davidson, ed.), Pergamon Press, 69-78, 1957.

LI, W. H., P. Bock, AND G. S. BeNroN, A new formus for flow into partially penetrating wells in aquaters, Trans. Amer. Geophys. Union, 35, 805-812, 1954.

Portman, D. J., Gier and Dunkle heat flow nxeters and heat storage variations, Exploring the Atwas phere's First Mile (H. Lettau and B. Davidson, ed. Pergamon Press, pp. 64-66, 1957.

RhoADEs, D. G., Gier and Dunkle heat flow maters Exploring the Atmosphere's First Mile (H. Lettax and B. Davidson, ed.), Pergamon Press, pp. $67-68$ 1957.

Universily of Michigan Research Institute, Ans An A Michigan

(Communicated manuscript received April 15, 1958.) 